\title{
Reciclado de desechos plásticos en Laboratorio de Manufactura
}

\author{
Recycling plastic waste in Manufacturing Laboratory
}

\author{
SÁNCHEZ-TORRES, Mónica Alejandra†*, GUZMAN-CORTÉS, Agustín y AGUILA-HERNÁNDEZ, \\ Llaned
}

Universidad Politécnica de Amozoc

ID $1^{\text {er }}$ Autor: Mónica Alejandra, Sánchez-Torres / ORC ID: 0000-0003-4301-4253, Researcher ID Thomson: Y-8188-2018, CVU CONACYT ID: 955325

ID $1^{\text {er }}$ Coautor: Agustín, Guzman-Cortés / ORC ID: 0000-0003-4571-224X Researcher ID Thomson: Y-2752-2018, CVU CONACYT ID: 222213

ID $2^{\text {do }}$ Coautor: Llaned, Aguila-Hernández / ORC ID: 0000-0003-2822-6565, CVU CONACYT ID: 351385

DOI: $10.35429 / J T I P .2019 .9 .3 .16 .23$

Recibido 26 de Octubre, 2019; Aceptado 14 Diciembre, 2019

\begin{abstract}
Resumen
México se encuentra en el primer lugar de países en producción de desechos plásticos; reciclando principalmente el polietilenotereftalato (PET), el reciclado de otros plásticos casi es nulo. Los equipos de laboratorios son elementos didácticos en la carrera de Ingeniería en Tecnologías de Manufactura; pues permite desarrollar competencias profesionales requeridas en dicha carrera, al integrar contenidos teóricos y experimentales a través de prácticas de laboratorio. El problema del uso continuo de las impresoras $3 \mathrm{D}$ en las prácticas es que, por cada kilogramo de ácido poliláctico (PLA) y acrilonitrilo butadieno estireno (ABS), resulta entre el $10 \%$ y $30 \%$ material para desecho. Difícilmente la universidad puede abastecer de estos insumos al Laboratorio de Manufactura por el alto costo de los materiales plásticos. Es por eso que el presente proyecto, busca establecer una propuesta tecnológica para darle una segunda vida a los desechos plásticos, producto de las actividades de las impresoras 3D de los laboratorios; disminuyendo el impacto ambiental generado por la universidad. Se pueden reciclar estos desechos, a través de método mecánico, y utilizarse como material en la realización de prácticas de laboratorio que, desarrollen competencias académicas y profesionales de nuevas tecnologías de Manufactura
\end{abstract}

Plásticos, Reciclado, Manufactura

\begin{abstract}
Mexico is ranned first place among countries with plastic waste production; recycling mainly polyethylene terephthalate (PET), the recycling of other plastics is almost nil. Laboratory equipment are didactic elements in Manufacturing Engineering; It allows to develop professional competences required in this major, by integrating theoretical and experimental contents through laboratory practices. The problem of continuous use of 3D printers in lab practices is that, for each kilogram of polylactic acid (PLA) and acrylonitrile butadiene styrene (ABS), between $10 \%$ and $30 \%$ results in waste material. The university can hardly afford these supplies to the Manufacturing Laboratory because of the high cost of plasctic materials. That is why this project seeks to offer a technological proposal to give plastic waste a second life, as an outcome from laboratory $3 \mathrm{D}$ printers. This may decrease the environmental impact generated by the university. These wastes can be recycled, through a mechanical method, and used as material in the realization of laboratory practices that develop academic and professional skills of new manufacturing technologies.
\end{abstract}

Plastics, Recycling, Manufacturing

Citación: SÁNCHEZ-TORRES, Mónica Alejandra, GUZMAN-CORTÉS, Agustín y AGUILA-HERNÁNDEZ, Llaned. Reciclado de desechos plásticos en Laboratorio de Manufactura. Revista de Tecnologías en Procesos Industriales. 2019 3-9: 16-23

\footnotetext{
* Correspondencia del Autor (Correo electrónico: monica.sanchez@upamozoc.edu.mx)

$\uparrow$ Investigador contribuyendo como primer autor.
} 


\section{Introducción}

El rápido aumento de la población mundial, ha demandado con la misma intensidad de bienes materiales para satisfacer sus necesidades, al grado de sustituir al metal, la madera, las fibras naturales, el papel, vidrios y porcelanas por elementos plásticos; convirtiendo al plástico en el material idóneo de manufactura, por económico y versátil. La demanda de productos plásticos inicio una economía primordial, ya que logro proveer a los consumidores de artículos domésticos, de oficina, ornamentales, ropa, juguetes, herramientas de trabajo, partes y piezas automotrices, envases y demás. De igual manera los plásticos superaron las barreras de uso particular, pues se incluyeron en actividades como, la industrial, en la construcción, en los transportes y las comunicaciones.

Otras ventajas de los plásticos, aparte de económicos es que, son ligeros, duraderos y se pueden manufacturar fácilmente en una variedad de productos, debido a sus excelentes propiedades como son: "baja densidad que permite la fabricación de objetos ligeros y reducción de costes de transporte; baja conductividad térmica y eléctrica; moldeo fácil, para adquirir formas variadas que sean rígidas o flexibles; gran resistencia a la corrosión y a la degradación" (Rivera, 2004, p. 3). Aunque al principio de la producción masiva de los plásticos, la calidad era bastante deficiente y mejoró hasta los años cincuenta, junto con la idea de crear nuevos productos versátiles, con mejor diseño y un modo más eficiente (Góngora, 2014).

La segunda revolución de los plásticos y quizá la más significativa para nuestra época, fue la "additive layer manufacturing", también conocida como impresión 3D. La impresión 3D inicio con la idea de crear mini y prototipos reales, y así poder valorar: medidas, funcionalidad, los procesos adecuados de manufactura y ensamblaje, anticipando todos estos aspectos a la etapa de producción industrial. Actualmente se puede imprimir en 3D cualquier cosa, con una creación desde cero a través de diseños digitales descargados de Internet como: herramientas para el hogar, estructuras para dispositivos electrónicos, reemplazos y sustituciones de piezas de plástico de aparatos, instrumentos de cuerda o viento y prototipos de todo tipo (Soto, 2014); hasta viviendas urbanas.
“En el ámbito médico, las prótesis hechas a medida cada vez son más populares, mientras se sigue investigando otras aplicaciones" (Miranda, 2017, párr. 4).

Como se mencionó, el uso de las impresoras 3D permite elaborar cualquier cosa teniendo el diseño, y es de gran utilidad para el análisis en los prototipos, previo a la producción en serie. El uso de los plásticos a través de las impresoras 3D, no sólo se quedó en el sector empresarial, sino que, trascendió hasta las aulas escolares. Según Elvira Rach "La digitalización y la tecnología están cambiando todo" (3Dnatives, 2018, párr. 6). Ya que, la impresión $3 \mathrm{D}$ se ha convertido en una necesidad profesional para la industria, es necesario que, la educación debe estar involucrada para formar estudiantes preparados para el futuro, por medio de la experiencia de aprendizaje, la enseñanza para los medios digitales y la integración de una diversidad de materias escolares para simplificar contenidos teóricos que resultan complejos de enseñar (3Dnatives, 2018).

\section{Planteamiento del problema}

La universidad Politécnica de Amozoc (UPAM) cuenta con un Laboratorio de Manufactura Avanzada altamente equipado; donde se realizan prácticas de laboratorio, propuestas en los manuales de asignatura que, conforman el mapa curricular de Ingeniería en Tecnologías de Manufactura (ITM). Dicha carrera tiene el propósito de dotar de habilidades y competencias al alumnado, a través de la simulación de los procesos de manufactura más comunes en la industria. El laboratorio de Manufactura avanzada está provisto entre otros equipos, con dos impresoras 3D profesionales $\mathrm{y}$ una inyectora de plásticos de mesa; disponibles para realizar un prototipado rápido y piezas en general, como parte de las prácticas de laboratorio en materias como: Ciencia e Ingeniería de los Materiales, Ingeniería de Plásticos, Tecnologías de Soporte en diseño y Manufactura, y Diseño para Manufactura y Ensamble.

La elaboración de las piezas y prototipos tienen como base el ensayo y error, así que es necesario que, en ocasiones se tengan que imprimir más de una vez hasta que, queden como lo solicitan las especificaciones de la práctica o proyecto. 
Aun cuando las piezas tienen las especificaciones requeridas, las impresoras ocasionalmente presentan un fallo, afectando la elaboración (Figura 1.). Estas causas principalmente, generan la mayor cantidad de desechos plásticos en el Laboratorio de Manufactura. Christian Lölkes (¿A quién dañan las impresoras 3D?, (s, f)), afirma que entre el 5 y 10 por ciento del total de los productos de impresión 3D, se desechan a la basura. Tan sólo la Universidad de Berkeley, la cual dispone de unas 100 impresoras 3D en el campus; generan más de 200 kilos anuales de residuos plásticos (González, s. f).

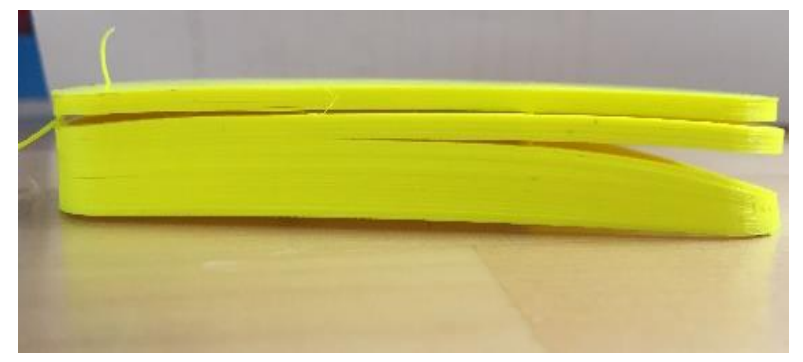

Figura 1 Pieza defectuosa del extremo derecho

Se realizó un cálculo, sobre la cantidad de desperdicios plásticos, producto de las impresoras 3D del Laboratorio de Manufactura y se estima que, la quinta parte del material utilizado en las impresiones se desecha; es decir, un kilogramo de plástico por cada cinco kilogramos de materia prima, se va a la basura. Aunque la UPAM tiene un sistema de separación de residuos, por medio de botes clasificados dentro y fuera de los laboratorios; las piezas defectuosas generadas en la impresión 3D, se han desechado directamente en los botes; ya que no se ha implementado una estrategia para después de su "uso".

Los desperdicios plásticos se pueden traducir en dinero; en el mercado, dependiendo del tipo material y características del mismo, el kilogramo de ABS PLA o el poliuretano termoplástico TPU de características sencillas ronda entre los \$250.00 y los \$950.00 MX; los materiales con características más complejas como brillantina, madera o metal por ejemplo tiene un valor entre $\$ 1000.00$ y $\$ 2500.00 \mathrm{MX}$.

\section{Justificación}

El uso de los plásticos tiene un desarrollo prometedor y asombroso sin duda alguna; pero lo que no es nada seguro, son los alcances que tendrán los plásticos después de su uso.
Únicamente tenemos evidencia de que, los plásticos han llegado a cada rincón del planeta, y no sólo de la tierra, también de lecho marino (Mckie, 2016); poniendo en serio peligro la biodiversidad que conocemos (SEMARNAT 2018, p. 20). Debido a que los plásticos sólo se han producido en masa durante unos 60 años, no se ha determinado con certeza su longevidad en el medio ambiente (Hopewell, Dvorak y Kosior, 2009).

$\mathrm{Si}$ bien ya existen acciones para disminuir la huella de los plásticos, como campañas contra bolsas, empaques y plásticos de un sólo uso, así como la creación de industrias dedicadas al reciclado plástico; siguen haciendo falta muchas acciones para disminuir la cantidad de plásticos, desechados a los vertederos.

Los plásticos, por su origen y composición, derivados del petróleo, considerado como materia prima agotable; "son un residuo de alto valor, relativamente fácil de recuperar y abundante (tanto o más que el vidrio en los residuos domésticos y creciente entre los residuos industriales)" (Arandes, Bilbao y López, 2004, p. 29).

Aunque México, es líder del reciclaje en América porque transforma el $58 \%$ del plástico utilizado, principalmente PET (Ramos, 2019); después se encuentran plásticos como el Polietileno (PE), Polietileno de alta densidad (PEAD), Policloruro de vinilo (PVC), Polietileno de baja densidad (PEBD, Polipropileno (PP), Poliestireno (PS); pero se desconocen los datos sobre la cantidad de desechos plásticos de ABS, PLA o TPU, y mucho menos se tiene información sobre el reciclado de estos materiales.

"La Ocean Conservancy señala que el reto mayor no es limpiar ..., sino evitar que el plástico siga llegando en masa" (SEMARNAT, 2018, p. 20).

Es importante tomar conciencia como académicos responsables y transmitir a los estudiantes, sobre la reutilización de los desechos plásticos que se generan en laboratorios y talleres, para que estos no lleguen a los vertederos, se quemen o sean enterrados como cualquier otro desperdicio. 
El reciclado de plásticos del Laboratorio de Manufactura, además brinda la oportunidad de proveer de forma alternativa material plástico a las prácticas de laboratorio, aumentar actividades académicas que están condicionadas por el uso limitado de materiales, reforzar contenidos teóricos del mapa curricular de ITM, disminuir el coste en la compra de insumos plásticos, aplicar y desarrollar nuevas tecnologías de manufactura y la posibilidad de abrir nuevos campos laborales.

\section{Objetivo}

Desarrollar e implementar una metodología para la recuperación y reciclaje de materiales plásticos en el Laboratorio de Manufactura de la UPAM.

\section{Objetivos secundarios}

- Disminuir la cantidad de desechos
plásticos que llegan a la basura.
Ahorrar en el costo de consumibles para
impresora 3D, para prácticas de
Laboratorio.
Reciclar y suministrar materiales
plásticos, para el Laboratorio de
Manufactura UPAM
Estudiar nuevos campos laborales
tecnológicos viables, eficientes y
económicos para materiales plásticos.

\section{Metodología a desarrollar para el reciclaje mecánico}

Se sabe que, durante los últimos años se han iniciado trabajos para reciclar polímeros, de las cuales destacan las siguientes: el procedimiento mecánico, el cual incluye trabajo manual o de máquinas; el procedimiento químico se basa en la descomposición del polímero en monómeros, dependiendo la pureza de estos se vuelven a incorporar en la fabricación de nuevos plásticos; el procedimiento energético consiste en la incineración de plásticos, para recuperar energía, aunque este tiene un alto impacto a la contaminación atmosférica (Arandes et al., 2004; Gaggino, 2008). Si bien es cierto que, en el mercado ya existe una variedad de equipos para trabajar el reciclaje de plásticos, desde lavadoras de material, molinos, trituradores, prensas y peletizadoras; pero, lo que no es nada accesible de estos equipos, es el precio.
Es por eso que, el actual trabajo presenta una metodología, basada en el reciclaje mecánico, el cual consta de tres etapas prácticas y la cuarta etapa queda como propuesta de seguimiento.

\section{Acopio y clasificación}

Se colocaron contenedores rotulados, para que se depositaran en ellos, las partes plásticas defectuosas o las consideradas como residuos para ser desechados. Cada contenedor está rotulado de acuerdo, al tipo de polímero que se utiliza dentro del laboratorio o puedan traer del exterior de la universidad (Figura 2.) y con los que se piensa trabajar en el presente proyecto.

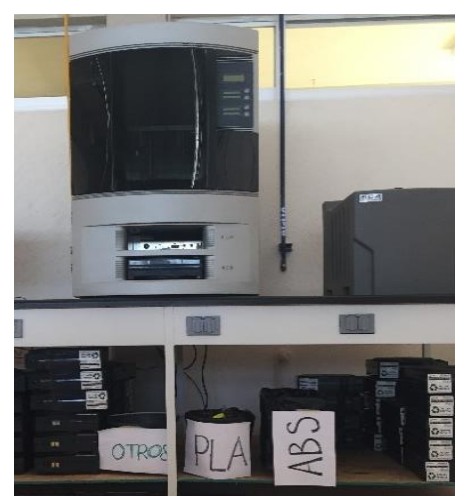

Figura 2 Contenedores rotulados, colocados debajo de las impresoras

\section{Trituración}

El proceso de trituración se llevó a cabo en una trituradora artesanal como se muestra en la figura 3; este mecanismo fue elaborado por alumnos de tercer cuatrimestre grupo uno, dentro de la materia de Fundamentos de la Electricidad. La trituradora tiene la finalidad de disgregar los termoplásticos de desecho en piezas pequeñas, para después pasar el material molido en un tamizador de $5 \mathrm{~mm}$ de abertura. Los desechos plásticos deben triturarse de acuerdo con su clasificación para no ser mezcladas entre sí y no se afecten las pruebas posteriores. Después del triturado se almacenaron en recipientes rotulados y sellados hasta su uso.
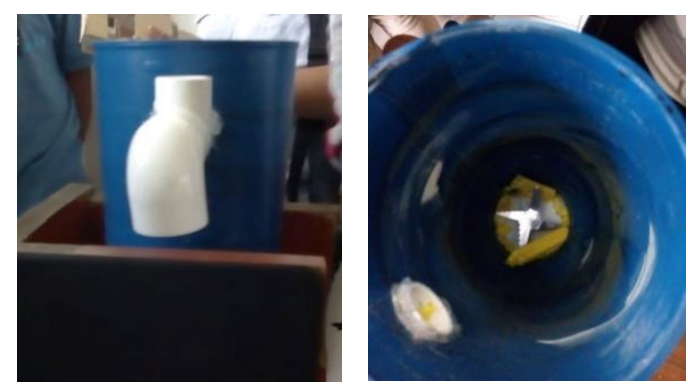

Figura 3 y 4 Trituradora artesanal utilizada

SÁNCHEZ-TORRES, Mónica Alejandra, GUZMAN-CORTÉS, Agustín y AGUILA-HERNÁNDEZ, Llaned. Reciclado de desechos plásticos en Laboratorio de Manufactura. Revista de Tecnologías en Procesos Industriales. 2019 

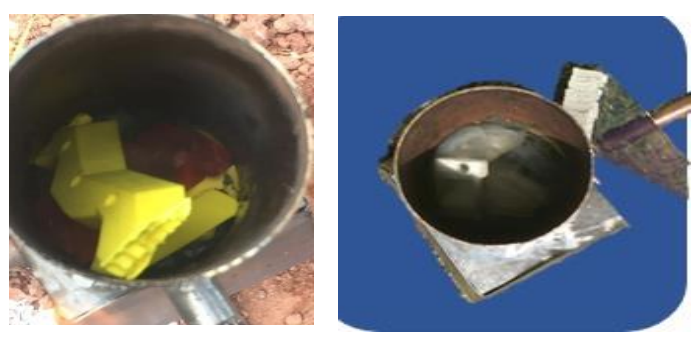

Figura 5 Pruebas de fundición para ABS y PLA

\section{Fundición}

Se fundió el material recolectado, el objetivo de este proceso es determinar la temperatura de fusión de cada material para considerar si se puede reutilizar dicho material. La prueba de fundición se realizó en un pequeño horno de acero (Figura 4.); éste fue elaborado por alumnos de tercer cuatrimestre grupo uno. El horno se calienta por medio de una hornilla de gas como se muestra del lado derecho de la Figura 4.

En el proceso de fundición se ocupó el horno elaborado para fundir por separado $300 \mathrm{~g}$ de ABS, $300 \mathrm{~g}$ de PLA (material triturado); para las piezas de TPU y la pieza completa de resina SLA debido a que no se contaba con la cantidad suficiente, y complicaba las maniobras en el horno, se optó por fundir este material utilizando un crisol y una parrilla eléctrica. Para cada prueba por separado, se mantuvo la hornilla encendida hasta alcanzar el punto de fusión de cada material (Figura 5.). Para los materiales de ABS y PLA se propuso igualar el color del material fundido. Durante el proceso de fundición se puede agregar colorante universal para polímeros en tono negro.

\section{Diseño de extrusora}
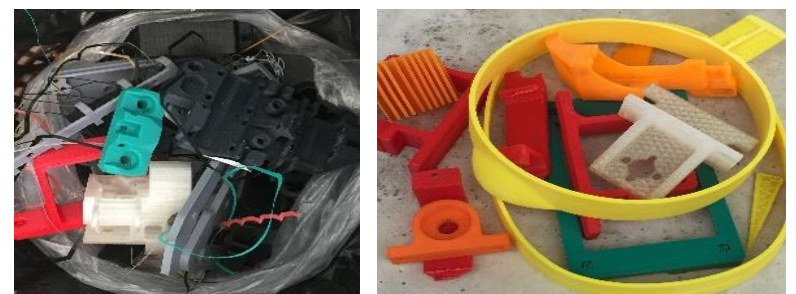

Figura 7 Piezas recolectadas de PLA (izquierda) y ABS (derecha)

Para un mejor aprovechamiento de los materiales plásticos a través del reciclado, la extrusión puede ser una alternativa como última etapa. Ya que, con esta propuesta se puede suministrar filamentos plásticos que se pueden reutilizar en impresoras 3D.
La Figura 6 muestran el diseño planteado para la elaboración de una extrusora con monohusillo, la cual cuenta con tres secciones elementales: alimentación, compresión y dosificación. El diseño de la extrusora se compone del husillo o tornillo será de acero inoxidable y filete helicoidal con una longitud de $20 \mathrm{~cm}$ de longitud y $1 \mathrm{~cm}$ de diámetro; un cilindro o barril será de acero inoxidable con una longitud de $22 \mathrm{~cm}$, una tolva corta con capacidad de $200 \mathrm{ml}$, con una garganta de alimentación de $2 \mathrm{~cm}$, y motor eléctrico para operar el tornillo. Este diseño se puede adaptar a los equipos propuestos en los procesos antes mencionados y completar el círculo del reciclado plástico de las impresoras 3D.

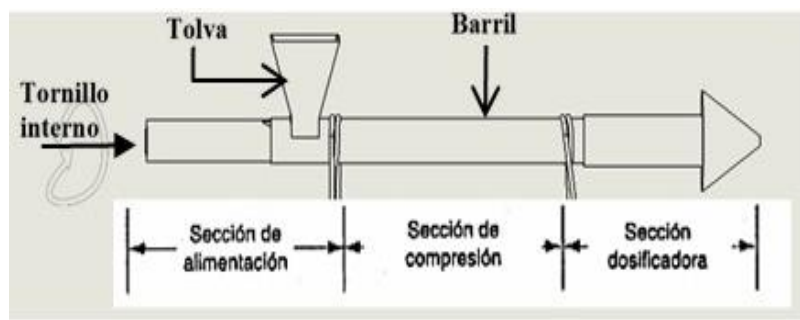

Figura 6 Diseño propuesto de extrusora

\section{Resultados}

Se logró recolectar $1253 \mathrm{~g}$ de PLA, $2720 \mathrm{~g}$ de ABS (Figura 7.), aproximadamente $95 \mathrm{~g}$ de TPU en unas almohadillas defectuosas para mouse (Figura 8.) y una pieza de resina de estereolitografía, por sus siglas en inglés SLA, de aproximadamente $50 \mathrm{~g}$ (Figura 9.). Aunque estos dos últimos materiales no se tenían contemplados para el proyecto, se mencionan ya que fueron colocados dentro del contenedor de "otros" materiales.

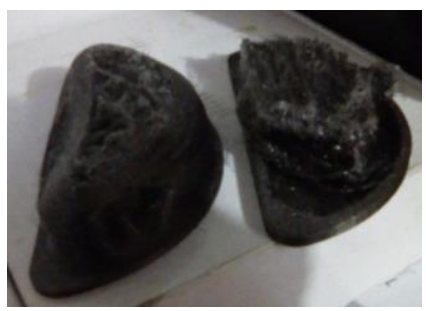

Figura 8 Almohadillas para mouse defectuosas de TPU

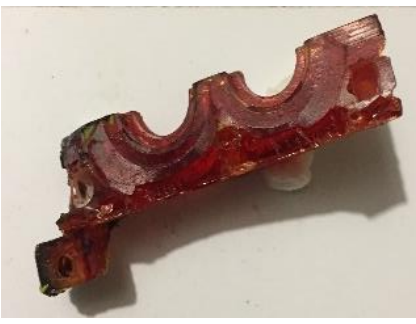

Figura 9 Pieza de resina SLA

SÁNCHEZ-TORRES, Mónica Alejandra, GUZMAN-CORTÉS, Agustín y AGUILA-HERNÁNDEZ, Llaned. Reciclado de desechos plásticos en Laboratorio de Manufactura. Revista de Tecnologías en Procesos Industriales. 2019 
Para las pruebas de trituración de ABS y PLA, no se mostraron dificultades, estas fueron trituradas en su totalidad y almacenadas por separado (Figura 10.). El material TPU no mostró dificultad en la trituración; sin embargo, la pieza de resina SLA no pudo ser procesada en la trituradora, ya que es una pieza muy compacta y la trituradora no tuvo efecto en ella, por lo que se decidió, dejar completa la pieza para la etapa de fundición.
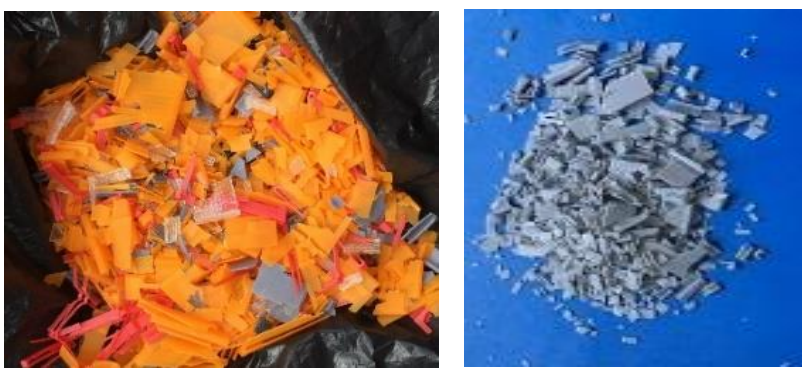

Figura 10 ABS (izquierda) y PLA (derecha) triturados

Para la prueba de fusión del TPU y de la pieza de resina SLA, se utilizó un crisol y una parrilla eléctrica; ya que la cantidad de muestra disponible de cada uno, era mínima, para utilizar el horno. Para el TPU en el proceso de fundición, no se logró el objetivo utilizando la parrilla eléctrica y el crisol. Este material alcanzo casi los $142{ }^{\circ} \mathrm{C}$ y únicamente se fundió la parte inferior que estaba en contacto con el crisol (Figura 11.), la apariencia del material del fondo era viscoso (Figura 12.), mientras que la parte que sobresalía de crisol mantuvo su forma sólida.

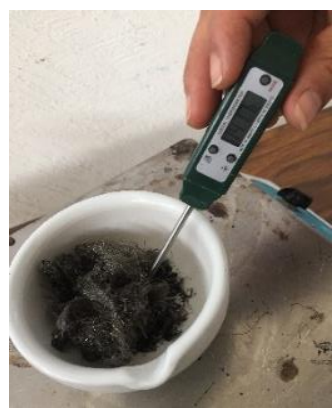

Figura 11 Fundición de TPU

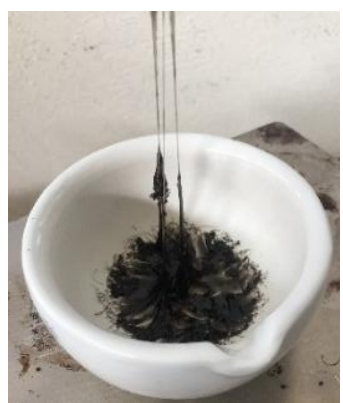

Figura 12 TPU semi fundido
A pesar de que el material TPU no logro fundirse en su totalidad, utilizando el crisol y la parrilla eléctrica; pusimos a prueba la pieza de resina SLA, con estas mismas condiciones. Pero la prueba de fundición para la resina SLA también resultó negativa, pues la parrilla eléctrica no alcanzó a suministrar el calor suficiente para derretir la pieza. Por lo que se optó por utilizar el horno para tratar de fundir la pieza. La pieza de resina SLA en el horno alcanzo $\operatorname{los} 240^{\circ} \mathrm{C}$, pero tampoco se pudo fundir por este método, quedando la pieza intacta (Figura 13.).

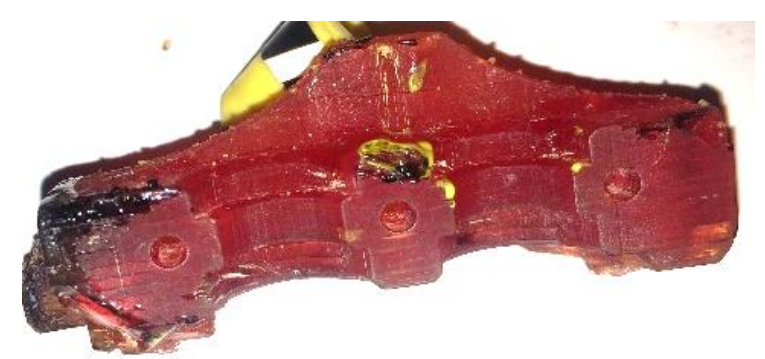

Figura 13 Pieza de resina SLA intacta después de pruebas de fundición

El proceso de fundición de ABS y PLA no mostró complicaciones utilizando el horno de fundición. Para estos materiales se realizaron tres pruebas de fusión a cada uno, con el propósito de obtener el rango de temperatura aproximada de fundición de cada uno. El rango de temperaturas alcanzadas se muestra en la Tabla 1, junto con los datos de las pruebas de fusión que se realizaron al TPU y la pieza de resina SLA.

\begin{tabular}{|l|l|}
\hline \multicolumn{2}{|c|}{ Material plástico } \\
\hline ABS & $210-230$ \\
\hline TPU & 142 aprox. \\
\hline PLA & $220-230$ \\
\hline Resina SLA & N/A \\
\hline
\end{tabular}

Tabla 1 Tabla de temperatura de fusión alcanzada por cada material

Debido a que el proceso de fundición de PLA y ABS, resultó adecuado utilizando el horno, en cuanto se logró la fundición total del material se procedió a peletizarlo de manera rudimentaria. Para peletizar, se dejó fluir el material líquido por el ducto de salida del horno, mientras fluía hacía un recipiente con agua para su enfriamiento, el flujo plástico se seccionó utilizando unas pinzas. 
Posterior a conocer las temperaturas de fusión del PLA, TPU y ABS y tener el material de ABS y PLA fundido y en forma de unidades, estos dos últimos se utilizaron como material de suministro en la inyectora de plásticos de mesa que se encuentra en el área de laboratorios de la UPAM (Figura 14.). La inyectora de mesa cuenta con dos moldes propios, uno es, un llavero con forma elíptica y el otro es un molde para el mango de una herramienta, como un desarmador. El objetivo de esta práctica, es verificar que se pueda reutilizar el plástico reciclado, producto de las impresoras 3D, en un equipo que también utiliza insumos plásticos. Se realizaron únicamente pruebas con $\mathrm{ABS}$ reciclado en la inyectora de mesa, utilizando como referencia el rango de temperaturas de fundición (Tabla 1).

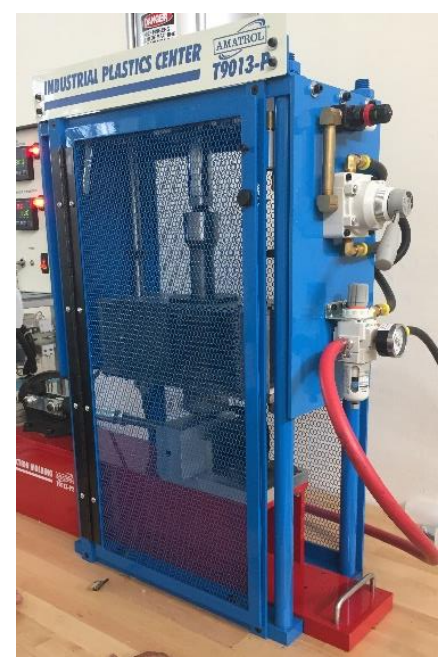

Figura 14 Inyectora de plásticos de mesa

Las prácticas realizadas en la inyectora, con el ABS reciclado mostraron resultados favorables en las piezas inyectas (Figuras $15 \mathrm{y}$ 16.). Estas piezas se formaron por completo dentro del molde, lo que significa que el material se fundió adecuadamente con la temperatura programada. Otra característica es que ninguna de las piezas tuvo grietas ni burbujas por aire en el interior del plástico. La pieza en forma de mago de herramienta, tiene una cohesión y dureza sólida (Figura 17.). Mientras que la pieza del llavero también mantiene una cohesión y firmeza constante en toda la estructura de la pieza.

Como resultado inherente se realizaron prácticas de laboratorio en las materias de Ingeniería de los Materiales, Química Básica y Procesos Secundarios de Manufactura, abordando temas con implicaciones importantes sobre el tratamiento y manufactura de polímeros.

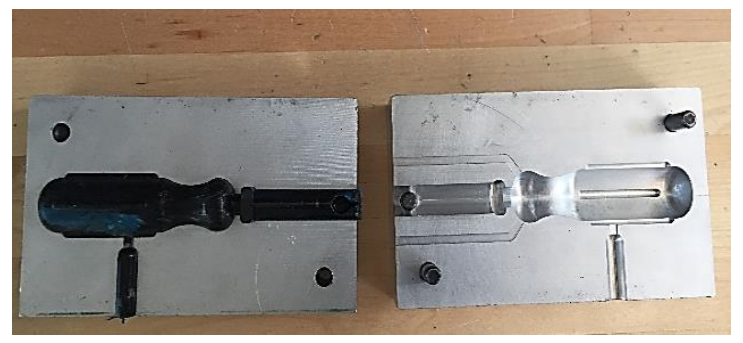

Figura 15 Pieza mango-herramienta inyectado con ABS reciclado

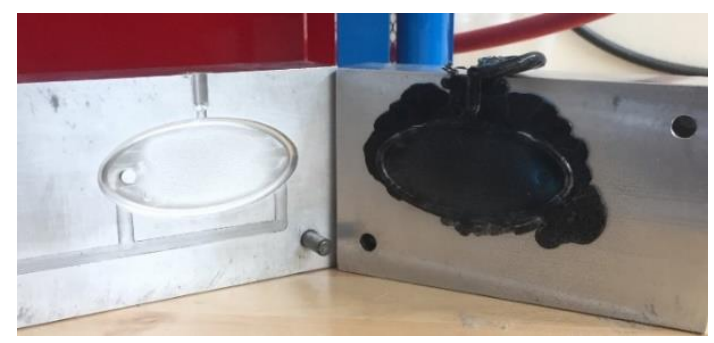

Figura 16 Pieza llavero inyectado con ABS reciclado
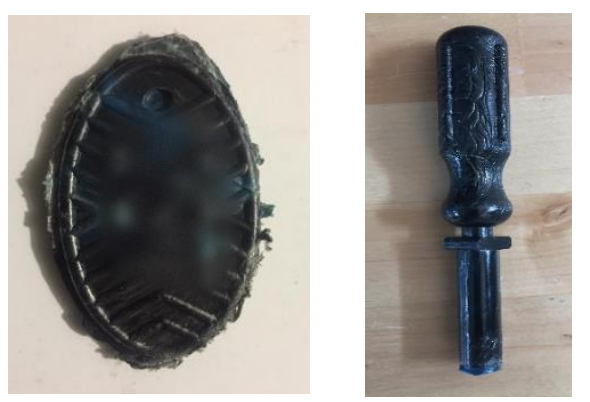

Figura 17 Piezas con buena cohesión, sin grietas ni burbujas de aire

\section{Conclusiones}

Con base en los resultados se puede concluir que la propuesta metodológica del presente proyecto, sólo resulta viable para reciclar desechos plásticos de ABS y PLA. Los materiales como el TPU y la resina SLA, no mostraron un trabajo de reciclaje factible desde el principio; ya que sólo se acopió una cantidad mínima de estos materiales, y aunque el TPU pasó la prueba de la trituradora y la resina SLA no; la confirmación que no es posible reciclar por el método mecánico estos dos materiales, es que ninguno, pudo superar la prueba de fundición. La restrictiva de este proceso, anula todas las posibilidades de formar un nuevo elemento plástico que, pueda ser utilizado o aplicado en algún otro proceso del Laboratorio de Manufactura Avanzada de la UPAM.

Mientras que el ABS y PLA, mostraron un comportamiento aceptable durante el proceso del reciclaje mecánico, propuesto en la metodología del presente proyecto. 
Concluyendo que tanto el ABS y el PLA son viables para reciclar por este método, y que el producto obtenido del reciclaje se puede emplear en otros equipos que requieren insumos plásticos. No será necesario invertir en su totalidad en materiales vírgenes para uso de estos equipos; ya que, la generación de desechos plásticos, producto principalmente de las impresoras 3D, es excesiva y en la misma medida, puede proveer material plástico reciclado, a través del método mecánico. Este proyecto no sólo ha beneficiado a la economía del Laboratorio, sino que, le ha dado sentido a la adquisición de conocimientos a través de la realización de prácticas de laboratorio, las cuales no se realizaban por falta de insumos plásticos.

Lo más importante en proporcionarle un segundo uso a los materiales plásticos de desecho, es que todo ese volumen a de plástico ya, no llagara a los vertederos, para acumularse por una eternidad. Este tipo de resultados despiertan la expectación, de seguir buscando nuevas tecnologías de manufactura, para procesar y minimizar los residuos sólidos que desechamos los humanos, en nuestro andar por este mundo industrializado que cambia con rapidez. Obedeciendo la Ley de Conservación de la materia que dice que, no se crea ni se destruye, sólo se transforma; no nos queda más que reciclar.

\section{Referencias}

3Dnatives el sitio web de la impresión 3D. (2018). Implementación de la impresión $3 D$ en la educación ¿una necesidad? Recuperado de https://www.3dnatives.com/es/impresion-3den-la-educacion-290820182/

¿A quién dañan las impresoras 3D? (s, f). En NATIONAL GEOGRAPHIC EN ESPAÑOL. Recuperado de https://www.ngenespanol.com/ ciencia/ciencia-impacto-ambiental-impresora3d-tercera-dimension/

Arandes, J. M., Bilbao, J. y López, D. (2004). Reciclado de residuos plásticos. En Revista Iberoamericana de Polímeros.

Gaggino, R. (2008). Ladrillos y placas prefabricadas con plásticos reciclados aptos para la autoconstrucción. En Revista INVI. Recuperado de http://revistainvi.uchile.cl/index.php/INVI/articl e/view/446/955
Góngora, J. P. (2014). La industria del plástico en México y el mundo. En Revistas Bancomext. Recuperado de http://revistas.bancomext.gob.mx/rce/magazine s/761/3/la_industria_del_plastico.pdf

González, V. (s, f). ¿Qué hacemos con los residuos de las impresoras 3D? En Revista Muy Interesante. Recuperado de https://www.muyinteresante.es/tecnologia/artic ulo/que-hacemos-con-los-residuos-de-lasimpresoras-3d-231487753280

Hopewell, J., Dvorak, R. y Kosior, E. (2009). Plastics recycling: challenges and opportunities. En The Royal Society Publishing. Recuperado de https://doi.org/10.1098/rstb.2008.0311

McKie, R. (2016). Plastic now pollutes every corner of Earth. En The Observer. Recuperado de

https://www.theguardian.com/environment/201 6/jan/24/plastic-new-epoch-human-damage

Miranda, I. (2017). Impresión en 3D, «la cuarta revolución». Recuperado de https://www.abc.es/sociedad/abci-impresioncuarta-revolucion-201706142127_noticia.html

Ramos, J. L., (2019, 9 de febrero). México, campeón de reciclaje. El Sol de México. Recuperado de https://www.elsoldemexico.com.mx/finanzas/re ciclaje-ecoce-botellas-de-plastico-3034337.html

Rivera, R. (2004). Propuesta de reciclaje mecánicoe plásticos en la ciudad de Piura. Universidad de Piura. Recuperado de https://pirhua.udep.edu.pe/bitstream/handle/110 42/1180/ING_418.pdf?sequence $=1$

SEMARNAT. (2018). Plástico, amenaza en los mares. (Boletín informativo). En NUESTRO Ambiente. Océanos y Mares son responsabilidad de todos.

Soto, J. A. (2014). 3D REV: una posible revolución de la producción empresarial. Universidad Pontificia Comillas. Recuperado de https://repositorio.comillas.edu/rest/bitstreams/ 182/retrieve. 